\title{
Hadronic transitions in bottomonia at Belle
}

\author{
Simon Eidelman ${ }^{1,2,3, *}$ \\ for the Belle Collaboration \\ ${ }^{1}$ Budker Institute of Nuclear Physics SB RAS, Novosibirsk, Russia \\ ${ }^{2}$ Novosibirsk State University, Novosibirsk, Russia \\ ${ }^{3}$ Lebedev Physical Institute RAS, Moscow, Russia
}

\begin{abstract}
Recent Belle results on various hadronic transitions among bottomonia are presented. We observe transitions with the $\eta$ and $\eta^{\prime}$ meson at the $\Upsilon(4 S)$. Bottomonium production together with the $\eta$ meson is also reported at the $\Upsilon(10860)$. We study $\pi^{+} \pi^{-} \pi^{0}$ transitions at the $\Upsilon(10860)$ and $\Upsilon(11020)$.
\end{abstract}

\section{Introduction}

Spectroscopy of heavy quarkonia provides crucial information for understanding strong interactions since QCD calculations become possible. Measurements of hadronic transitions $\left(\pi^{+} \pi^{-}, \eta, \omega, \ldots\right)$ between bottomonia yield important input for QCD. $\eta$ transitions are believed to be suppressed compared to $\pi^{+} \pi^{-}$because of the spin flip. $\pi^{+} \pi^{-}$transitions and their peculiarities were studied by both BaBar and Belle. Large integrated luminosity collected by Belle above the $\Upsilon(4 S)$ opened unique possibilities resulting in exciting observations of $h_{b}(1 P), h_{b}(2 P), \eta_{b}(2 S), Z_{b}(10610)$ and $Z_{b}(10650)$. In this work we discuss recent results on hadronic transitions in bottomonia.

\section{Study of $\eta$ and $\pi^{+} \pi^{-}$transitions in $\Upsilon(4 S)$ decays to lower $b \bar{b}$}

Here we study the transitions $\Upsilon(4 S) \rightarrow \pi^{+} \pi^{-} \Upsilon(n S)(n=1,2)$ and $\Upsilon(4 S) \rightarrow \eta \Upsilon(1 S)$, by reconstructing $\Upsilon(n S) \rightarrow \mu^{+} \mu^{-}, \eta \rightarrow \pi^{+} \pi^{-} \pi^{0}$ using (538 \pm 7) $\times 10^{6} \Upsilon(4 S)$ mesons [1].

For the dipion transitions, the two-dimensional distribution of the invariant dimuon mass $M(\mu \mu)$ vs. $\Delta M$ for the selected events is shown in Fig. 1 , where $\Delta M=M(\pi \pi \mu \mu)-M(\mu \mu)$. The signal yields are determined from the fit to the $\Delta M$ distribution in $6 \times 4(4 \times 4)$ bins of $M\left(\pi^{+} \pi^{-}\right)$and $\cos \theta_{\text {hel }}\left(\pi^{+}\right)$in the regions (a) and (b) due to the initial-state radiation processes $\Upsilon(2 S) \rightarrow \pi^{+} \pi^{-} \Upsilon(1 S)$ and $\Upsilon(3 S) \rightarrow \pi^{+} \pi^{-} \Upsilon(1 S)$, respectively (in (c) and (d) due to the decays of interest $\Upsilon(4 S) \rightarrow \pi^{+} \pi^{-} \Upsilon(1 S)$ and $\Upsilon(4 S) \rightarrow \pi^{+} \pi^{-} \Upsilon(2 S)$, respectively). The results are listed in Table 1 and the $\Delta M$ distributions integrated over the bins are shown in Fig. 2.

For the $\eta$ transitions, the distributions of $\Delta M_{\eta}=M(\pi \pi \gamma \mu \mu)-M(\mu \mu)-M(\pi \pi \gamma \gamma)$ are shown in Fig. 3 for the $\Upsilon(4 S) \rightarrow \eta \Upsilon(1 S)$ (left) and $\Upsilon\left(1^{3} D_{1,2}\right) \rightarrow \eta \Upsilon(1 S)$ (right).

All the results are compatible with the previous measurements with the slightly improved precision. We confirm the enhancement of the $\Upsilon(4 S) \rightarrow \Upsilon(1 S)$ transition via the spin-flip exchange of the $\eta$ meson with respect to the dipion one.

\footnotetext{
*e-mail: simon.eidelman@ cern.ch
} 


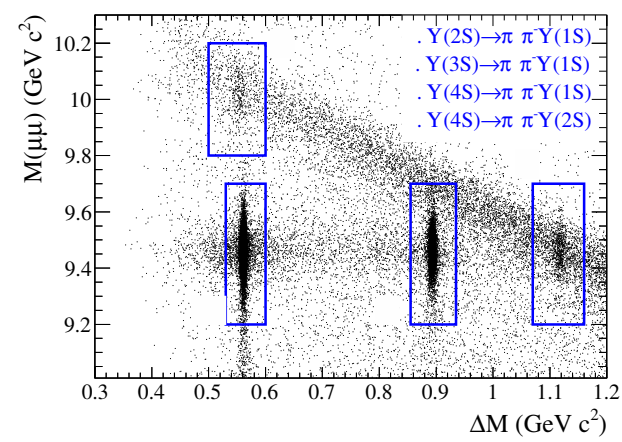

Figure 1. Distribution of $M(\mu \mu)$ vs. $\Delta M$ for the selected events. Fit regions are enclosed in boxes.
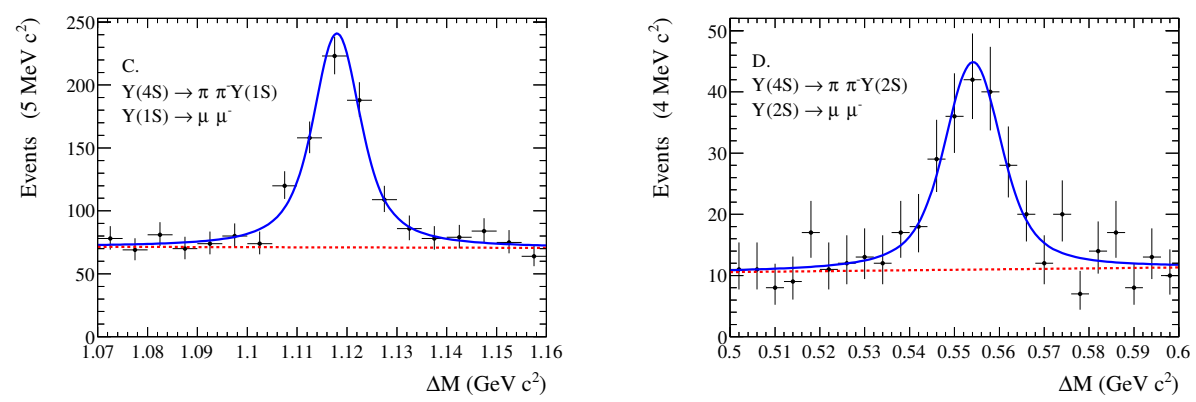

Figure 2. Fits to the $\Delta M$ distributions for: (left) $\Upsilon(4 S) \rightarrow \pi^{+} \pi^{-} \Upsilon(1 S)$ and (right) $\Upsilon(4 S) \rightarrow \pi^{+} \pi^{-} \Upsilon(2 S)$.

Table 1. The signal yields and branching fractions compared to PDG [2] for the decay modes studied.

\begin{tabular}{lccc}
\hline Decay & Events & $\mathcal{B}, 10^{-5}$ & $\mathcal{B}_{\mathrm{PDG}}, 10^{-5}$ \\
\hline$\Upsilon(4 S) \rightarrow \pi^{+} \pi^{-} \Upsilon(1 S)$ & $1095 \pm 74$ & $8.2 \pm 0.5 \pm 0.4$ & $8.1 \pm 0.6$ \\
$\Upsilon(4 S) \rightarrow \pi^{+} \pi^{-} \Upsilon(2 S)$ & $821 \pm 107$ & $7.9 \pm 1.0 \pm 0.4$ & $8.6 \pm 1.3$ \\
$\Upsilon(4 S) \rightarrow \eta \Upsilon(1 S)$ & $49 \pm 7$ & $1.70 \pm 0.23 \pm 0.08$ & $1.96 \pm 0.28$ \\
$\Upsilon\left(1^{3} D_{1,2}\right) \rightarrow \eta \Upsilon(1 S)$ & $2.1 \pm 3.0$ & $<0.23$ & - \\
\hline
\end{tabular}

\section{Observation of $\Upsilon(4 S) \rightarrow \eta^{\prime} \Upsilon(1 S)$}

This observation is based on the same data sample of $(538 \pm 7) \times 10^{6} \Upsilon(4 S)$ mesons as in Sect. 2. We reconstruct $\Upsilon(1 S) \rightarrow \mu^{+} \mu^{-}$and $\eta^{\prime} \rightarrow \rho \gamma, \eta \pi^{+} \pi^{-}, \eta \rightarrow 2 \gamma$ [3].

Signal events are identified by the variable $\Delta M_{\eta^{\prime}}=M(\Upsilon(4 S))-M(\Upsilon(1 S))-M\left(\eta^{\prime}\right)$, where $M(\Upsilon(1 S))=M(\mu \mu), M(\Upsilon(4 S))=M(\mu \mu \pi \pi \gamma)[M(\mu \mu \pi \pi \gamma \gamma)]$ and $M\left(\eta^{\prime}\right)=$ $M(\pi \pi \gamma)[M(\pi \pi \gamma \gamma)]$. The signal and background yields are obtained by the fit to the $\Delta M_{\eta^{\prime}}$ distributions shown in Fig. 4 and are also listed in Table 2.

The combined branching fraction is $\mathcal{B}\left(\Upsilon(4 S) \rightarrow \eta^{\prime} \Upsilon(1 S)\right)=(3.43 \pm 0.88 \pm 0.21) \times 10^{-5}$ and corresponds to the $5.7 \sigma$ statistical significance.

We also determine the ratios of branching fractions:

$$
R_{\eta^{\prime} / h}=\frac{\mathcal{B}\left(\Upsilon(4 S) \rightarrow \eta^{\prime} \Upsilon(1 S)\right)}{\mathcal{B}(\Upsilon(4 S) \rightarrow h \Upsilon(1 S))}
$$


Table 2. The signal and background yields as well as significances for two $\eta^{\prime}$ decay modes.

\begin{tabular}{lccc}
\hline$\eta^{\prime}$ decay mode & $N_{\text {sig }}$ & $N_{\text {bkg }}$ & Sign., $\sigma$ \\
\hline $2 \pi 1 \gamma$ & $22 \pm 7$ & $96 \pm 11$ & 4.2 \\
$2 \pi 2 \gamma$ & $5.0 \pm 2.3$ & $2.0 \pm 1.6$ & 4.1 \\
\hline
\end{tabular}

where the decay is mediated by $h=\eta$ or $h=\pi^{+} \pi^{-}$and using the values from our work [1] obtain $R_{\eta^{\prime} / \eta}=0.20 \pm 0.06$ and $R_{\eta^{\prime} / \pi^{+} \pi^{-}}=0.42 \pm 0.11$. The former ratio agrees with the predicted 0.2 [4].

\section{Observation of $\Upsilon(4 S) \rightarrow \eta h_{b}(1 P)$}

From a sample of $771.6 \times 10^{6} \Upsilon(4 S)$ decays $\left(711 \mathrm{fb}^{-1}\right)$ Belle observed for the first time the decay $\Upsilon(4 S) \rightarrow \eta h_{b}(1 P)$ using the $\eta$ missing mass, $M_{\text {miss }}(\eta)=\sqrt{\left(P_{e^{+} e^{-}}-P_{\eta}\right)^{2}}$ and $\eta \rightarrow$ $\gamma \gamma$ [5]. The $M_{\text {miss }}(\eta)$ distribution is shown in Fig. 5 (left). The clear $h_{b}(1 P)$ signal is observed with the fit yield of $112469 \pm 5537$ events $(11 \sigma$ significance $)$.

The obtained branching fraction $\mathcal{B}\left(\Upsilon(4 S) \rightarrow \eta h_{b}(1 P)\right)=(2.18 \pm 0.11 \pm 0.18) \times 10^{-3}$ agrees with the theoretical prediction of Ref. [6].

We reconstruct the $h_{b}(1 P) \rightarrow \gamma \eta_{b}(1 S)$ decay by measuring the number of events of $\Upsilon(4 S)$ decay as a function of the variable $\Delta M_{\text {miss }}=M_{\text {miss }}(\eta \gamma)-M_{\text {miss }}(\eta)$. Its distribution (Fig. 5 (right)) shows a clear excess at $\Delta M_{\text {miss }}=M_{\eta_{b}(1 S)}-M_{h_{b}(1 P)}$, with a yield of $33116 \pm 4741$ events $\left(9 \sigma\right.$ significance). The corresponding branching fraction $\mathcal{B}\left(h_{b}(1 P) \rightarrow \gamma \eta_{b}(1 S)\right)=$ $(56 \pm 8 \pm 4) \%$ agrees with predictions [7,8]. The results are summarized in Table 3 .

Table 3. Summary of the searches for $\Upsilon(4 S) \rightarrow \eta h_{b}(1 P)$ and $h_{b}(1 P) \rightarrow \gamma \eta_{b}(1 S)$.

\begin{tabular}{lc}
\hline Observable & Value, $\mathrm{MeV}$ \\
\hline$M_{h_{b}(1 P)}$ & $9899.3 \pm 0.4 \pm 1.0$ \\
$M_{\eta_{b}(1 S)}$ & $9400.7 \pm 1.7 \pm 1.6$ \\
$\Gamma_{\eta_{b}(1 S)}$ & $8_{-5}^{+6} \pm 5$ \\
$\Delta M_{\mathrm{HF}}(1 P)=M_{\chi_{b J}}^{\mathrm{sa}}(1 P)-M_{h_{b}(1 P)}$ & $+0.6 \pm 0.4 \pm 1.0$ \\
$\Delta M_{\mathrm{HF}}(1 S)=M_{\Upsilon(1 S)}-M_{\eta_{b}(1 S)}$ & $59.6 \pm 1.7 \pm 1.6$ \\
\hline
\end{tabular}
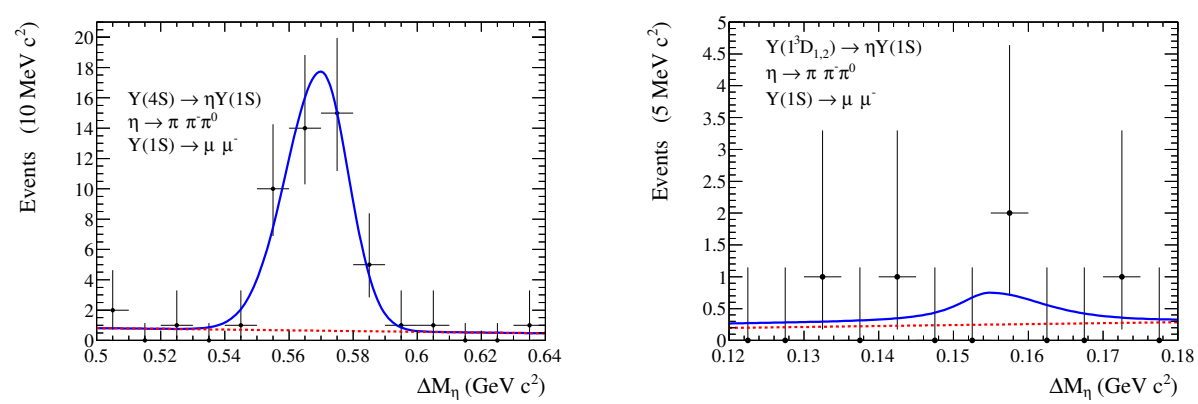

Figure 3. Fits to the $\Delta M_{\eta}$ distributions for: (left) $\Upsilon(4 S) \rightarrow \eta \Upsilon(1 S)$ and (right) $\Upsilon\left(1^{3} D_{1,2}\right) \rightarrow \eta \Upsilon(1 S)$. 

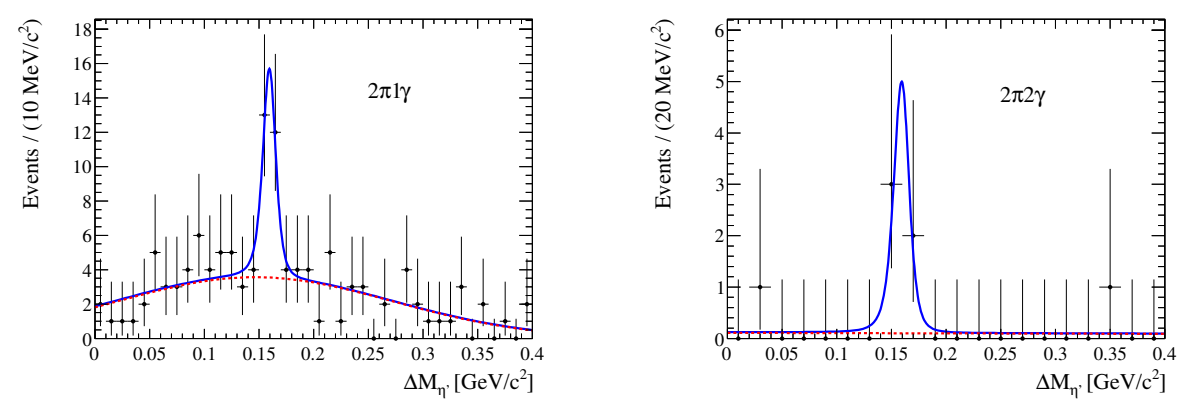

Figure 4. Fit to the $\Delta M_{\eta^{\prime}}$ distribution for $\Upsilon(4 S) \rightarrow \eta^{\prime} \Upsilon(1 S)$ candidates in the $2 \pi 1 \gamma$ (left) and $2 \pi 2 \gamma$ (right) modes.
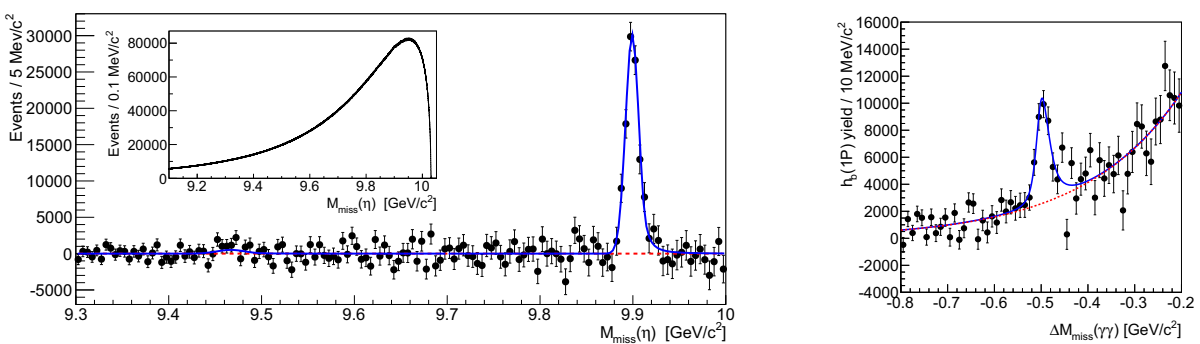

Figure 5. Distributions of: (left) $M_{\text {miss }}(\eta)$ after background subtraction, (right) $\Delta M_{\text {miss }}$.

\section{Production of $b \bar{b}$ and $\eta$ near $\Upsilon(5 S)$}

For this study we used a sample of $121.4 \mathrm{fb}^{-1}$ collected near the $\Upsilon(5 S)$ energy [9]. The approach is based on the missing mass $M_{\text {miss }}(\eta)$ similar to that in Sect. 4. The corresponding distribution is shown in Fig. 6 with the results listed in Table 4.

Table 4. Summary of the studies of $e^{+} e^{-} \rightarrow \eta(b \bar{b})$ near $\Upsilon(5 S)$.

\begin{tabular}{lccccc}
\hline Process & Sign., $\sigma$ & $N, 10^{3}$ & Process & Sign., $\sigma$ & $N, 10^{3}$ \\
\hline$\eta \Upsilon(1 S)$ & $1.5 \sigma$ & $1.7 \pm 1.0$ & $\eta \Upsilon(2 S)$ & $3.3 \sigma$ & $5.6 \pm 1.6$ \\
$\eta h_{b}(1 P)$ & $2.7 \sigma$ & $3.9 \pm 1.5$ & $\eta \Upsilon(1 D)$ & $5.3 \sigma$ & $9.3 \pm 1.8$ \\
\hline
\end{tabular}

We observe for the first time the process $e^{+} e^{-} \rightarrow \eta \Upsilon_{J}(1 D)$ and find evidence for $e^{+} e^{-} \rightarrow$ $\eta \Upsilon(2 S)$. Assuming that the process proceeds entirely via the $\Upsilon(5 S)$ and taking $\sigma\left(e^{+} e^{-} \rightarrow\right.$ $\Upsilon(5 S))=\sigma\left(e^{+} e^{-} \rightarrow b \bar{b}\right)=(0.340 \pm 0.016) \mathrm{nb}$ from our measurement [10], we obtain $\mathcal{B}\left(\Upsilon(5 S) \rightarrow \eta \Upsilon_{J}(1 D)\right)=(4.82 \pm 0.92 \pm 0.67) \times 10^{-3}$. This result agrees with theoretical estimates accounting for the effect of virtual $B$-meson loops [11]. We do not have significant evidence for $e^{+} e^{-} \rightarrow \eta h_{b}(1 P, 2 P)$ nor $e^{+} e^{-} \rightarrow \eta \Upsilon(1 S)$. We do not have direct evidence for the presence of the three states of the $\Upsilon_{J}(1 D)$ triplet and derive $90 \%$ CL upper limits on the fraction of $J=1$ and $J=3$ states with respect to the $J=2$ state. 


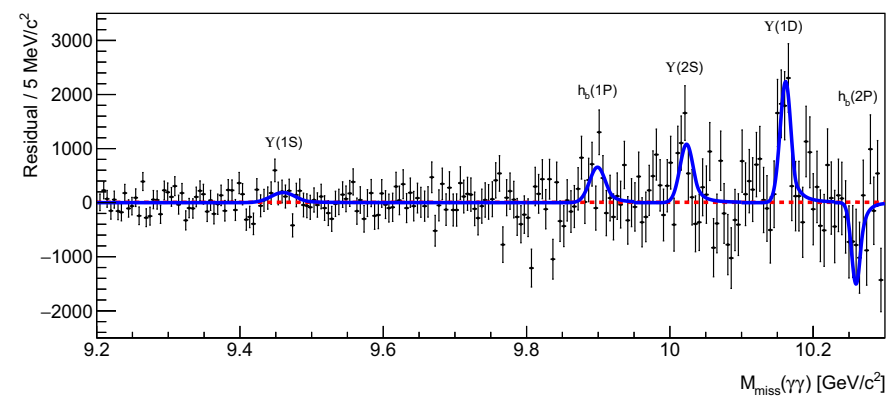

Figure 6. $M_{\text {miss }}(\eta)$ distribution after subtraction of the fitted background.

\section{Observation of $e^{+} e^{-} \rightarrow \pi^{+} \pi^{-} \pi^{0} \chi_{b J}$ at $10.867 \mathrm{GeV}$}

A data sample of $118 \mathrm{fb}^{-1}$ collected at $10.867 \mathrm{GeV}$ has been used to study $\pi^{+} \pi^{-} \pi^{0}$ transitions between the $\Upsilon(5 S)$ and $\chi_{b J}, J=0,1,2$, with subsequent $\chi_{b J} \rightarrow \gamma \Upsilon(1 S), \Upsilon(1 S) \rightarrow l^{+} l^{-}, l=$ $e, \mu$ decays [12].

Figure 7 shows the scatter plot of $M\left(\pi^{+} \pi^{-} \pi^{0}\right)$ vs. $M\left(\gamma^{\Upsilon} \Upsilon(1 S)\right)$. In addition to the clear $\omega$ signal in the $\chi_{b J}$ mass region, there is an obvious accumulation of $\left(\pi^{+} \pi^{-} \pi^{0}\right)_{\text {non- } \omega}$ events above the $\omega$ mass. A two-dimensional fit gives the $\omega \chi_{b J}$ and $\left(\pi^{+} \pi^{-} \pi^{0}\right)_{\text {non- } \omega \chi_{\text {bJ }}}$ yields. Figure 8 shows the $\pi^{+} \pi^{-} \pi^{0}$ mass projection (left) with the obvious $\omega$ signal and evidence for non- $\omega$ events, and the $\gamma^{\top} \Upsilon(1 S)$ mass projection within (center) and outside (right) the $\omega$ region with clear $\chi_{b 1}$ and $\chi_{b 2}$ signals. The fit results are summarized in Table 5. No excess of $\chi_{b 0}$ events above expected backgrounds is observed. The branching fractions of the three-pion transitions are large and of the same order as two-pion processes.

We also search for the $X$ (3872)-like state $X_{b}$ in the process $e^{+} e^{-} \rightarrow \gamma X_{b}, X_{b} \rightarrow \omega \Upsilon(1 S)$ and observe no excess of events over the expectation from the $\omega \chi_{b J}$ final states. The upper limit is $\mathcal{B}\left(\Upsilon(5 S) \rightarrow \gamma X_{b}\right) \mathcal{B}\left(X_{b} \rightarrow \omega \Upsilon(1 S)\right)<2.9 \times 10^{-5}$ at $90 \%$ CL.

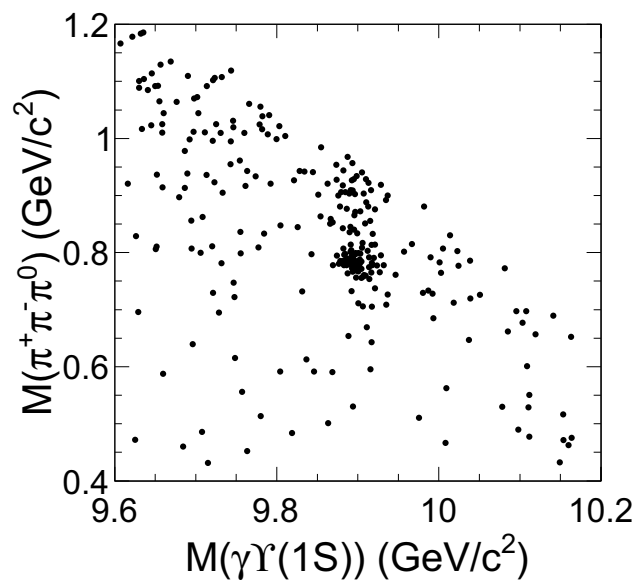

Figure 7. Scatter plot of $M\left(\pi^{+} \pi^{-} \pi^{0}\right)$ vs. $M(\gamma \Upsilon(1 S))$ for selected $e^{+} e^{-} \rightarrow \pi^{+} \pi^{-} \pi^{0} \gamma \Upsilon(1 S)$ events. 
Table 5. Results of the fits

\begin{tabular}{lccc}
\hline Mode & Yield & Sign., $(\sigma)$ & $\mathcal{B}, 10^{-3}$ \\
\hline $3 \pi \chi_{b 1}$ & $80.1 \pm 9.9$ & 12 & $1.85 \pm 0.23 \pm 0.23$ \\
$3 \pi \chi_{b 2}$ & $28.6 \pm 6.5$ & 5.9 & $1.17 \pm 0.27 \pm 0.14$ \\
\hline$\omega \chi_{b 1}$ & $59.9 \pm 8.3$ & 12 & $1.57 \pm 0.22 \pm 0.21$ \\
$\omega \chi_{b 2}$ & $12.9 \pm 4.8$ & 3.5 & $0.60 \pm 0.23 \pm 0.15$ \\
\hline$(3 \pi)_{\text {non }-\omega \chi_{b 1}}$ & $23.6 \pm 6.4$ & 4.9 & $0.52 \pm 0.15 \pm 0.11$ \\
$(3 \pi)_{\text {non }-\omega \chi_{b 2}}$ & $15.6 \pm 5.4$ & 3.1 & $0.61 \pm 0.22 \pm 0.28$ \\
\hline
\end{tabular}
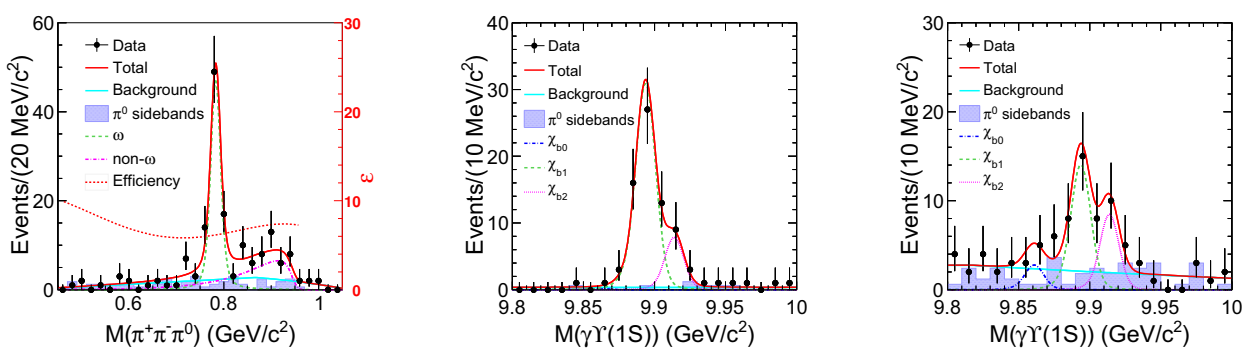

Figure 8. Projections of: (left) $M\left(\pi^{+} \pi^{-} \pi^{0}\right)$ for $9.8<M\left(\gamma^{\top} \Upsilon(1 S)\right)<10 \mathrm{GeV}$, (center) $M\left(\gamma^{\top} \Upsilon(1 S)\right)$ in the $\omega$ region, (right) $M(\gamma \Upsilon(1 S))$ outside of the $\omega$ region.

Finally, we search for $\Upsilon(6 S) \rightarrow \omega_{b J}, \phi \chi_{b J}$ using scan data near 10.867 and 11.020 $\mathrm{GeV}$ [13]. We observe $e^{+} e^{-} \rightarrow \pi^{+} \pi^{-} \pi^{0} \chi_{b 1}$ near $\Upsilon(6 S)$ and find evidence for $\omega \chi_{b J}$ while there is no significant signals for $\phi \chi_{b J}$ from 10.96 to $11.05 \mathrm{GeV}$.

We thank the KEKB group for excellent operation of the accelerator. This work was supported by Ministry of Science and Higher Education of the Russian Federation, contract 14.W03.31.0026.

\section{References}

[1] E. Guido et al., Phys. Rev. D 96, 052005 (2017)

[2] C. Patrignani et al., Chin. Phys. C 40, 100001 (2016)

[3] E. Guido et al., Phys. Rev. Lett. 121, 062001 (2018)

[4] M.B. Voloshin, Mod. Phys. Lett. A 26, 773 (2011)

[5] U. Tamponi et al., Phys. Rev. Lett. 115, 142001 (2015)

[6] F.-K. Guo, C. Hanhart and U.-G. Meissner, Phys. Rev. Lett. 105, 162001 (2010)

[7] S. Godfrey and J.L. Rosner, Phys. Rev. D 66, 014012 (2002)

[8] D.-Y. Chen, X. Liu and T. Matsuki, Phys. Rev. D 87, 094010 (2013)

[9] U. Tamponi et al., Eur. Phys. J. 78, 633 (2018)

[10] S. Esen et al., Phys. Rev. D 87, 031101 (2013)

[11] B. Wang, D.Y. Chen and X. Liu, Phys. Rev. D 94, 094039 (2016)

[12] X.H. He et al., Phys. Rev. Lett. 113, 142001 (2014)

[13] J.H. Yin et al., arXiv:1806.06203 